\title{
Particle Swarm and Population Structure
}

\author{
Carlos M. Fernandes \\ Agostinho C. Rosa \\ LARSyS: Laboratory for \\ Robotics and Systems in \\ Engineering and Science, \\ University of Lisbon, Portugal \\ cfernandes@laseeb.org
}

\author{
Nuno Fachada \\ HEI-LAB - Digital Human- \\ Environment and \\ Interactions Labs, \\ Universidade Lusófona, \\ Lisbon, Portugal \\ nfachada@laseeb.org
}

\author{
J.L.J. Laredo \\ LITIS, University of \\ Le Havre, Le Havre, \\ France \\ juanlu.jimenez@univ \\ -lehavre.fr
}

\author{
J.J. Merelo \\ Department of Computer \\ Architecture \\ University of Granada, \\ Granada, Spain \\ jmerelo@geneura.ugr.es
}

\begin{abstract}
We investigate the convergence speed, accuracy, robustness and scalability of PSOs structured by regular and random graphs with $3 \leq k \leq n$. The main conclusion is that regular and random graphs with the same averaged connectivity $k$ may result in significantly different performance, namely when $k$ is low.
\end{abstract}

Keywords: Particle Swarm Optimization, Population Structure.

\section{INTRODUCTION}

In the Particle Swarm Optimization (PSO) algorithm [1], information on the current and previous state of the search flows through the graph that connects the particles. The graph structure can be of any form and affects the balance between exploration and exploitation (and consequently, convergence speed and accuracy). Studies have tried to understand what makes a good structure. For instance, Kennedy and Mendes [2] investigated several types of topologies and recommend the use of a lattice with von Neumann. Others, like Parsopoulos and Vrahatis [3], have tried to design networks that hold the best traits given by each structure.

This paper revisits the study in [2]. Although the authors provided significant insight on the relationship between population structure and PSO performance, the study was mainly dedicated to random topologies and few levels of connectivity were inspected. Some aspects of the research that were overlooked are now worth investigating, namely the importance of graph regularity and the performance of regular and random graphs with the same level of connectivity.

\section{EXPERIMENTAL SETUP}

Several regular graphs have been constructed using the following procedure: starting from a ring structure with $(k=3)$ the degree is increased by linking each individual to its neighbors' neighbors, thus creating a set of regular graphs with $k=\{3,5,7,9,11 \ldots, n\}$, as exemplified in Figure 1 for a swarm with population size 7.

PSOs with population size $n=33$ have been used and regular graphs with $k=\{3,5,7,9,13,17,25,33\}$ were constructed. Then, random graphs with 33, 66, 99, 132, 198, 264 and 396 bidirectional edges were also generated, corresponding to an average level of connectivity $k^{\prime}=\{3,5,7,9,13,17,25,33\}$. Please

Permission to make digital or hard copies of part or all of this work for personal or classroom use is granted without fee provided that copies are not made or distributed for profit or commercial advantage and that copies bear this notice and the full citation on the first page. Copyrights for third-party components of this work must be honored. For all other uses, contact the Owner/Author.

GECCO '18 Companion, July 15-19, 2018, Kyoto, Japan

(C) 2018 Copyright is held by the owner/author(s).

ACM ISBN 978-1-4503-5764-7/18/07.

httns://doi.org/10.1145/3205651.3205779
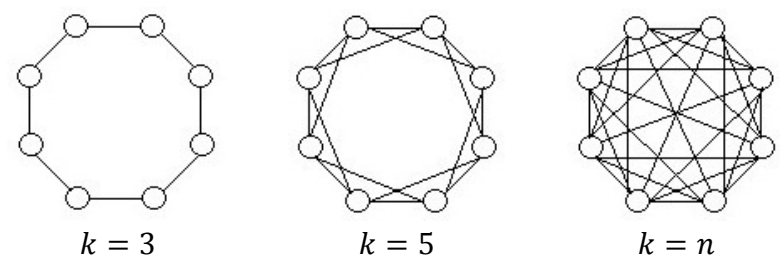

Figure 1. Regular graphs with population size $n=8$.

note that the regular graph with $k=33$ and the random graph with $k^{\prime}=33$ are equivalent to gbest structures. Acceleration coefficients were set to 1.49618 and inertia weight to 0.729844 . $X \max$ is defined as usual by the domain's upper limit and $V \max =X \max$. A total of 50 runs for each experiment were performed. Nine benchmark problems were used: functions $f_{1}-f_{3}$ are unimodal; $f_{4}-f_{7}$ are multimodal; $f_{8}$ is the shifted $f_{2}$ with noise and $f_{9}$ the rotated $f_{5}$. Asymmetrical initialization is used.

Two sets of experiments were conducted. First, the algorithms were run for a specific amount of function evaluations (330000 for $f_{1}$ and $f_{3}, 660000$ for the remaining). After each run, the best solution was recorded. Each algorithm has been executed 50 times in each function and statistical measures were taken over those runs. Then, the algorithms were all run for 660000 function evaluations or until reaching a function-specific stop criterion. A success measure was defined as the number of runs in which an algorithm attains the stop criterion. The algorithms discussed in this paper are available in the OpenPSO package available at https://github.com/laseeb/openpso.

\section{RESULTS AND DISCUSSION}

The first test compares success rates, convergence speed and accuracy on regular graphs. Problem dimension is $D=30$. Figure 2 shows the success rates on each function with each graph. In general, better rates are attained with lower connectivity. These results are in general terms in accordance with [2].

Figure 3 represents the evaluations (median values) required to meet the stop criteria. Clearly, convergence speed increases with connectivity $k$. These findings are different from those in [2], where it is reported that configurations with $k=5$ (from a set with $k=3, k=5$ and $k=10$ ) require less evaluations to meet the criteria. Table 1 shows the best fitness (median values) for each function and each graph. The best graphs according to the accuracy criteria depend on the type of function. For unimodal functions $\left(f_{1}, f_{2}, f_{3}\right.$ and $\left.f_{8}\right)$ best results are attained with highly connected graphs, while multimodal functions require lower connectivity. In [2], configurations with $k=5$ yielded the best fitness values and required less evaluations to meet the criteria, while $k=3$ had the best success rates. 


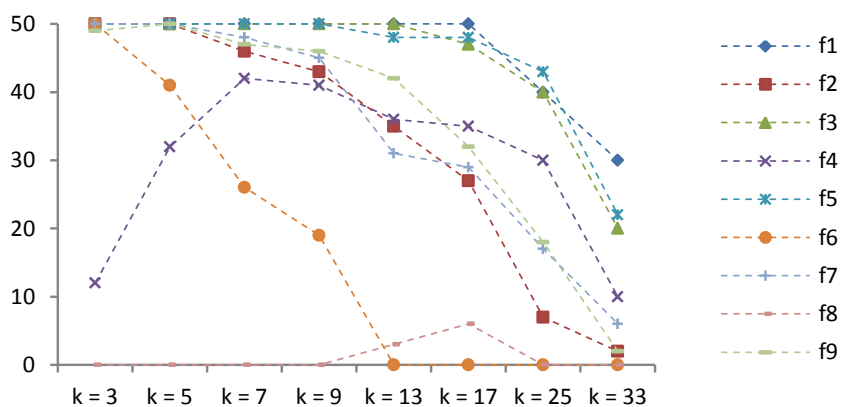

Figure 2. Success rates. Regular graphs.

The following test compares regular and random graphs with the same average connectivity. Figure 4 shows the success rates with different random graphs. When $k$ is low, PSO has much more difficulties in attaining the criteria. For higher $k$, the performance of regular and random graphs is more similar (see Figure 5).

This may be explained by the variance in the connectivity degree of random graphs: the standard deviation of $k$ in random graphs with low connectivity is high and decreases with $k$ (for $k=n$ the resulting graph is the gbest regular graph with null standard deviation of $k$ ). More tests are required but the main conclusion here is that for the same average $k$ it is preferable to use a regular graph (unless $k$ is close to $n$, in which case it is indifferent).

The fitness values were also affected by the randomness of lower connectivity structures: random graphs with lower $k$ clearly degrade the accuracy of PSO on many functions, namely in multimodal functions. As for the convergence speed, MannWhitney tests for comparing the distributions of the number of evaluations required by each graph in each function concluded than in the majority of the scenarios there are no significant differences in the ranking of the number of evaluations.

These experiments demonstrated that switching from a regular to a random graph with the same level of connectivity degrades PSO success rates and accuracy when $k$ is low, while for higher $k$ the results are similar. This is probably due to the high variance of the average $k$ in graphs with low connectivity but further investigation is required to confirm this hypothesis. However, the analysis in this paper has been mainly qualitative and supported by graphical depiction of the results. In the future, the data will be organized and normalized in order to perform exhaustive statistical tests that will hopefully give more insight on the relationship between performance and population structure.

Table 1. Best fitness. Median values. Regular graphs.

\begin{tabular}{|c|c|c|c|c|c|c|c|c|}
\hline & $k=3$ & $k=5$ & $k=7$ & $k=9$ & $k=13$ & $k=17$ & $k=25$ & $k=33$ \\
\hline 1 & $1.96 \mathrm{e}-89$ & 7.85e-90 & 3.93e-90 & $1.96 \mathrm{e}-90$ & $1.96 \mathrm{e}-90$ & $0.00 \mathrm{e} 00$ & $0.00 \mathrm{e} 00$ & 3.93e-90 \\
\hline$f_{2}$ & $7.59 \mathrm{e}-13$ & $1.04 \mathrm{e}-20$ & $2.49 \mathrm{E}-25$ & $4.41 \mathrm{e}-29$ & $3.03 e-34$ & 6.04e-37 & $1.00 e+04$ & $2.00 e+04$ \\
\hline 3 & $\mid 1.67 \mathrm{e}-88$ & 3.34e-89 & $5.89 e-90$ & $1.96 \mathrm{e}-90$ & $0.00 \mathrm{e} 00$ & $0.00 \mathrm{e} 00$ & $0.00 \mathrm{e} 00$ & $4.50 e+04$ \\
\hline$f_{4}$ & $1.18 e+02$ & $8.71 \mathrm{e}+01$ & $8.31 e+01$ & $7.26 e+01$ & $8.31 \mathrm{e}+01$ & $8.66 \mathrm{e}+01$ & $8.71 \mathrm{e}+01$ & $1.28 \mathrm{e}+02$ \\
\hline$f_{5}$ & $0.00 e+00$ & $0.00 e+00$ & $0.00 e+00$ & $0.00 e+00$ & $1.11 \mathrm{e}-02$ & 7.40e-03 & 9.86e-03 & $6.85 e-02$ \\
\hline$f_{6}$ & $0.00 e+00$ & $0.00 e+00$ & $6.17 \mathrm{e}-03$ & $6.78 \mathrm{e}-02$ & $1.02 e+00$ & $2.03 e+00$ & $4.33 e+00$ & $6.03 e+00$ \\
\hline 17 & $7.55 \mathrm{e}-15$ & 7.55e-15 & 7.55e-15 & 7.55e-15 & $7.55 e-15$ & $7.55 e-15$ & $1.25 e+00$ & $1.90 \mathrm{e}+00$ \\
\hline$f_{8}$ & $2.02 e+02$ & $1.32 e+01$ & 9.23e-01 & $3.43 e-01$ & $4.98 \mathrm{e}+03$ & $9.30 \mathrm{e}+03$ & $2.86 e+04$ & $4.74 \mathrm{e}+04$ \\
\hline & $0.00 \mathrm{e} 00$ & $0.00 \mathrm{e} 00$ & $0.00 \mathrm{e} 00$ & 8.63e-03 & $1.23 e-02$ & $1.72 \mathrm{e}-02$ & $5.09 \mathrm{e}-01$ & $4.25 e+01$ \\
\hline
\end{tabular}

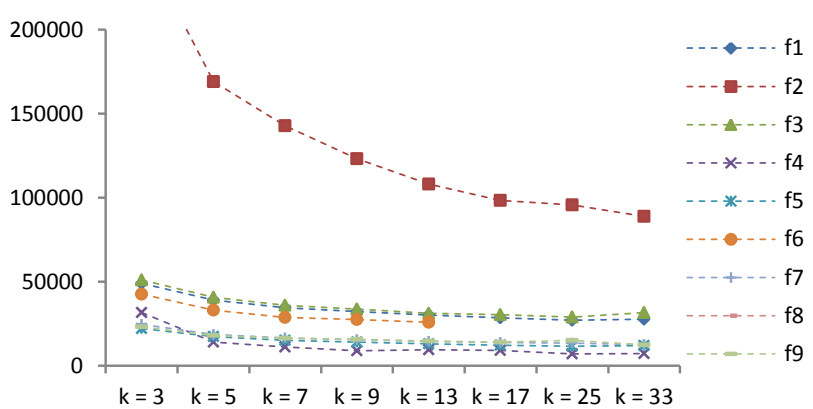

Figure 3. Evaluations. Regular graphs.

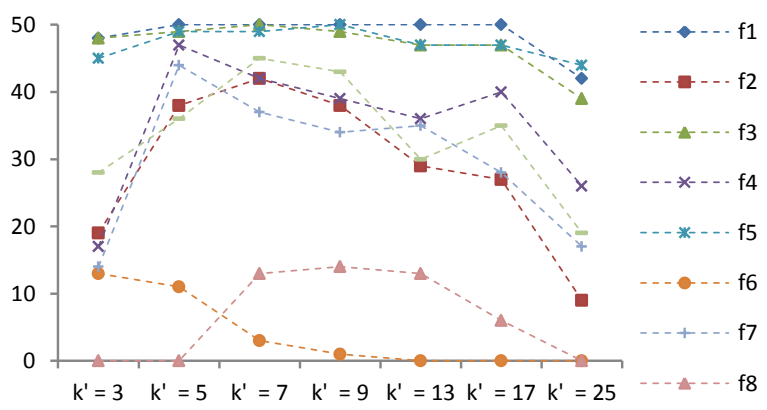

Figure 4. Success rates (50 runs). Random graphs.

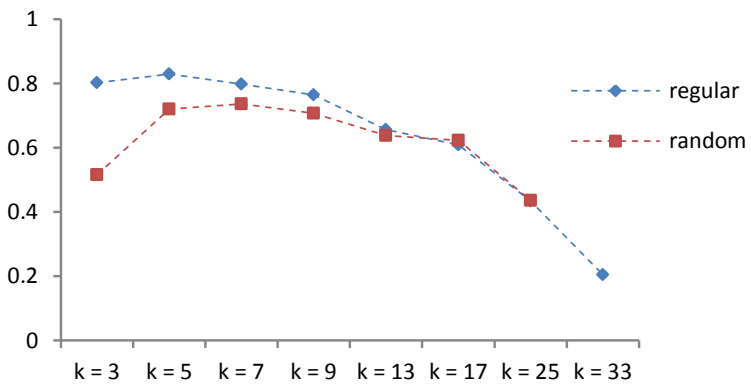

Figure 5. Percentage of successful runs averaged over the set of functions. Regular and random graphs.

\section{ACKNOWLEDGEMENTS}

First author wishes to thank FCT, Ministério da Ciência $e$ Tecnologia, his Fellowship SFRH/BPD/111065/2015). This work was supported by FCT PROJECT [UID/EEA/50009/2013].

\section{References}

[1] Kennedy, J., Eberhart, R. 1995. Particle Swarm Optimization. In Proceedings of IEEE International Conference on Neural Networks, Vol.4, 1942-1948.

[2] Kennedy, J., Mendes, R. 2002. Population structure and particle swarm performance. In Proceedings of the IEEE World Congress on Evolutionary Computation, 1671-1676.

[3] Parsopoulos, K.E., Vrahatis, M.N. 2005. Unified Particle Swarm Optimization in Dynamic Environments. Lecture Notes in Computer Science, Vol. 3449, Springer, 590-599. 